\title{
Direct Monitoring of Reconstructed Segmental Arterial Pressure during Deep Hypothermic Thoracoabdominal Aortic Aneurysm Surgery
}

\author{
Yuya Kise, MD, PhD, Yukio Kuniyoshi, MD, PhD, Mizuki Ando, MD, Tatuya Maeda, MD, \\ Hitoshi Inafuku, MD, PhD, and Satoshi Yamashiro, MD, PhD
}

\begin{abstract}
Deep hypothermia in thoracoabdominal aortic aneurysm operations is considered extremely useful for ensuring sufficient time to reconstruct the segmental arteries feeding the spinal cord. However, because the amplitude of motor evoked potentials (MEPs) decrease or disappear during deep hypothermia, feasible methods for assessing spinal cord circulation have not yet been reported. Performing additional segmental arterial reconstructions that rely on MEPs is also impractical. In the present case, to ascertain spinal cord circulation under deep hypothermia, we intraoperatively measured the reconstructed segmental arterial pressure in real time and investigated whether sufficient spinal cord blood flow had been attained.
\end{abstract}

Keywords: thoracoabdominal aortic aneurysm, segmental arterial pressure, deep hypothermia

\section{Introduction}

Methods for spinal cord protection during thoracoabdominal aortic aneurysm (TAAA) surgery remain under discussion, including methods for assessing spinal cord circulation during aortic cross-clamping. Motor evoked potentials (MEPs) are essential for ascertaining that cere-

Department of Thoracic and Cardiovascular Surgery, Graduate School of Medicine, University of the Ryukyus, Nishihara, Okinawa, Japan

Received: May 6, 2019; Accepted: June 26, 2019

Corresponding author: Yuya Kise, MD, PhD. Department of Thoracic and Cardiovascular Surgery, Graduate School of Medicine, University of the Ryukyus, 207 Uehara, Nishihara, Okinawa 903-0215, Japan

Tel: +81-98-895-1168, Fax: +81-98-895-1422

E-mail: yuya1022@med.u-ryukyu.ac.jp

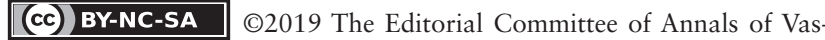
cular Diseases. This article is distributed under the terms of the Creative Commons Attribution License, which permits use, distribution, and reproduction in any medium, provided the credit of the original work, a link to the license, and indication of any change are properly given, and the original work is not used for commercial purposes. Remixed or transformed contributions must be distributed under the same license as the original. brospinal nerve pathways remain intact; therefore, they are used to detect spinal cord ischemia. ${ }^{1)}$ MEP amplitude decreases when the descending aorta is clamped, which decreases blood supply to the spinal cord through the segmental arteries. However, under deep hypothermia below $20^{\circ} \mathrm{C}$, MEPs usually disappear. ${ }^{2)}$ Therefore, evaluating circulation to the spinal cord during surgery is difficult under deep hypothermia, which is induced to avoid spinal cord ischemic injury (SCII). We recognize that assessing and maintaining spinal cord circulation before and during aortic cross-clamping are essential. In this report, we present an effective method for protecting the spinal cord by displaying the reconstructed segmental arterial pressure (rSAP), such as that of the artery of Adamkiewicz (AKA) and/or major segmental arteries (SAs), in real time. With this rSAP monitoring, we are able to estimate the spinal cord blood flow (SCBF) needed during aortic clamping to maintain adequate spinal cord perfusion.

\section{Case Report}

The patient was a 65 -year-old man scheduled to undergo surgery for Type V TAAA that had been diagnosed incidentally. Although the AKA was not visualized on preoperative 3-dimensional computed tomography, four pairs of large SAs at Th9-Th12, branching from the aneurysm, were detected (Figs. 1A and 1B).

As a surgical strategy, in an attempt to extend the ischemic tolerance time during aortic clamping and reconstruction of the major SAs, we planned aneurysm resection and prosthetic graft replacement, with upper and lower body perfusion, under deep hypothermia. In addition, SA reconstruction of the right intercostal artery was planned at the Th10 level, the center of the aneurysm.

After anesthesia induction, monitoring MEPs was initiated. Blood pressure (BP) was measured at the right radial artery and left femoral artery. The patient was placed in a right semi-lateral position. The aneurysm was approached through thoracotomy in the 8th intercostal space and retroperitoneal dissection. Taping around the aneurysm 


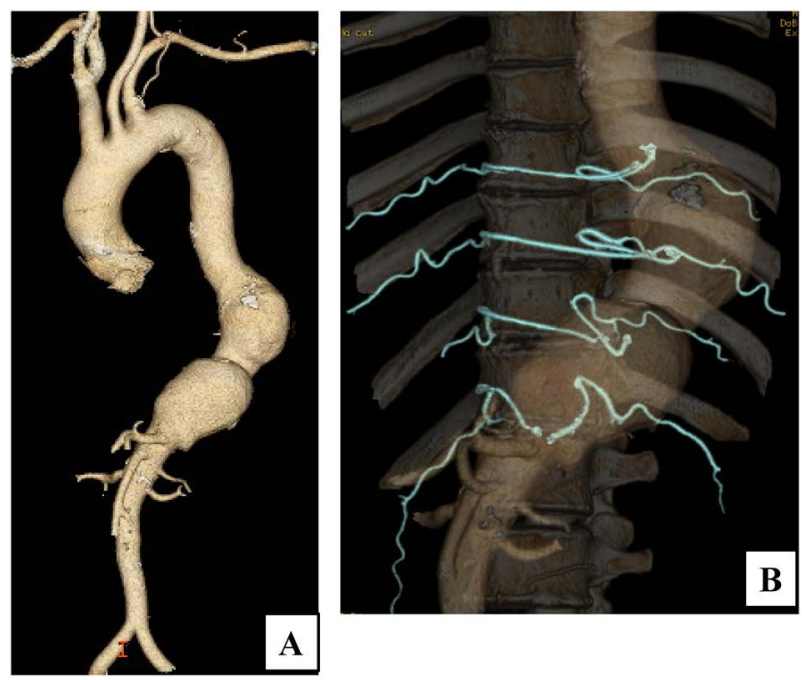

Fig. 1 (A) Preoperative 3-dimensional computed tomography examination shows two saccular aneurysms with a maximum diameter of $55 \mathrm{~mm}$. (B) Four pairs of thick segmental arteries are observed from the aortic aneurysm, but the artery of Adamkiewicz is not visualized.

was performed at Th8, just proximal to the superior mesenteric artery (SMA). After infusing 300 units $/ \mathrm{kg}$ of heparin, two arterial cannulations were performed at the Th7 descending thoracic aorta for perfusion of the upper body and right femoral artery (FA) (FEMII 16-Fr; Edwards Lifesciences, Irvine, CA, USA) for lower body perfusion. A long cannula for venous drainage was placed from the right femoral vein into the right atrium. Body temperature, as measured by tympanic temperature, was cooled to $20^{\circ} \mathrm{C}$. A left ventricle $(\mathrm{LV})$ venting tube was set from the LV apex to avoid over-dilatation of the LV. The amplitude of MEPs decreased with decreasing the body temperature. At a body temperature below $23^{\circ} \mathrm{C}$, all MEPs disappeared. At a body temperature of $20^{\circ} \mathrm{C}$, which was reached after more than $30 \mathrm{~min}$ of core cooling, the aorta at the Th8 level and the aneurysm just proximal to the SMA were clamped for proximal anastomosis, then opened (Fig. 2A). Backflow via collateral vessels was observed from the ostium of the four pairs of SAs at Th9-Th12. Among these, the right-sided Th10 intercostal artery was re-implanted because of the larger ostial size and the relatively high backflow volume. The remaining ostia were blocked to prevent a "vascular steal phenomenon" by the tourniquet technique, which consists of a purse string suture around the target ostium. The backflow of blood from the ostium of Th10 was blocked by a balloon catheter. With a 26-mm, size 4 branch prosthetic graft for TAAA (J-Graft SHIELD NEO; Japan Lifeline, Tokyo, Japan), proximal anastomosis was performed at the Th8 level of the aorta. Re-implantation of the Th10 right SA was performed with a $10-\mathrm{mm}$ graft prosthesis, anastomosed to a side branch

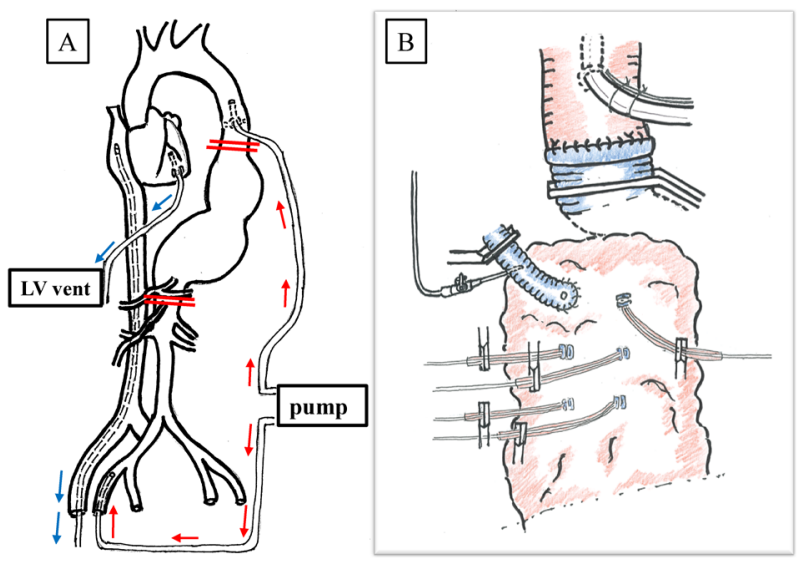

Fig. 2 (A) Schema of the perfusion technique. The aorta is crossclamped under deep hypothermia, simultaneous upper and lower body perfusion is continued, and collateral flow to the spinal cord is maintained. (B) After proximal anastomosis, the right segmental artery at the Th10 level is reconstructed under deep hypothermia, and reconstructed segmental arterial pressure via the collateral network is measured. LV: left ventricle

above the main graft. To assess reconstruction of the Th10 right SA, some variety of measurements under the following conditions were performed on completion of the anastomosis, as described above. The BP measured in the implanted graft prosthesis (Figs. 2B and 3A) is the rSAP, indicating the $\mathrm{BP}$ driving spinal cord circulation.

We measured rSAP under the following conditions to evaluate intraoperative spinal cord circulation (Fig. 3C).

\section{Condition 1}

The perfusion flow rate of the upper and lower body by cardiopulmonary bypass $(\mathrm{CPB})$ during aortic clamping was adjusted to $55 \mathrm{ml} / \mathrm{m} / \mathrm{kg}$. Noradrenaline (NOA) was continuously administered at $0.1 \mu \mathrm{g} / \mathrm{kg} / \mathrm{h}$, and mean BP was maintained at $70-80 \mathrm{mmHg}$ for the upper body and $80-90 \mathrm{mmHg}$ for the lower body. The rSAP was $50-55 \mathrm{mmHg}$ (approximately $60 \%-70 \%$ of pre-clamping systemic BP) and SCBF, via collateral flow, was similar to the pre-clamping value, indicating satisfactory spinal cord circulation.

\section{Condition 2}

The ostium of the SAs at Th9, left Th10, and Th11-12 that were blocked by the tourniquet, were opened (Fig. 3B). This led to decreased rSAP to $40 \mathrm{mmHg}$ (approximately $50 \%$ of pre-clamping systemic BP), due to the "vascular steal phenomenon." This verified the presence of a tight network between the reconstructed SA and the surrounding occluded SAs. 


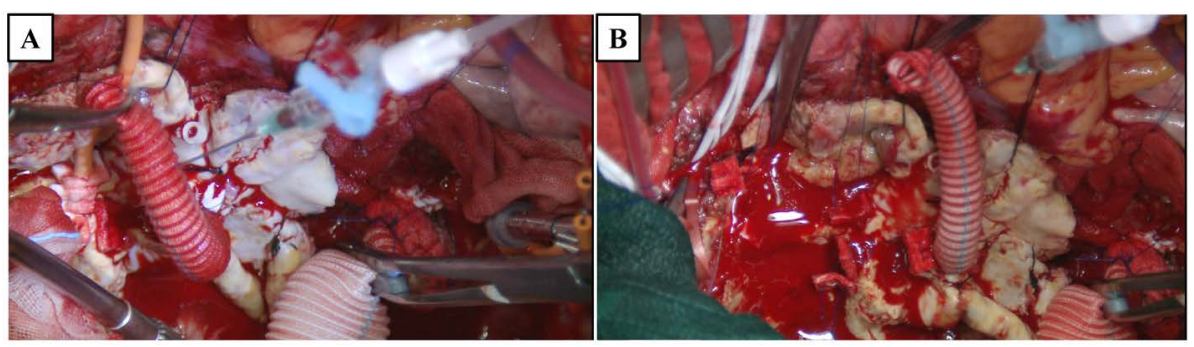

\begin{tabular}{|l|c|c|c|c|c|c|}
\hline C & $\begin{array}{c}\text { Pressure of } \\
\text { upper body } \\
(\mathrm{mmHg})\end{array}$ & $\begin{array}{c}\text { Pressure of } \\
\text { lower body } \\
(\mathrm{mmHg})\end{array}$ & $\begin{array}{c}\text { rSAP } \\
(\mathrm{mmHg})\end{array}$ & $\begin{array}{c}\text { Upper body } \\
\text { perfusion }\end{array}$ & $\begin{array}{c}\text { Lower body } \\
\text { perfusion }\end{array}$ & $\begin{array}{c}\text { Blood steal } \\
\text { from ostia of } \\
\text { intercostal } \\
\text { arteries }\end{array}$ \\
\hline Condition 1 & 79 & 98 & 53 & $(+)$ & $(+)$ & $(-)$ \\
\hline Condition 2 & 78 & 96 & $40 \downarrow$ & $(+)$ & $(+)$ & $(+)$ \\
\hline Condition 3 & 76 & $30 \downarrow$ & $30 \downarrow$ & $(+)$ & $(-)$ & $(-)$ \\
\hline Condition 4 & 73 & $87 \uparrow$ & $50 \uparrow$ & $(+)$ & $(+)$ & $(-)$ \\
\hline
\end{tabular}

Fig. 3 (A, B) Intraoperative photograph. (C) Monitoring of reconstructed segmental arterial pressure (rSAP).

(A) rSAP is measured (Condition 1). (B) The tourniquet of the surrounding segmental arteries is loosened, and rSAP is measured under a "vascular steal phenomenon" (Condition 2). (C) The change in rSAP under each condition is shown. Under Conditions 2 and 3 , marked decreases in rSAP are observed.

\section{Condition 3}

Lower body perfusion from the right FA was stopped, and lower body pressure was decreased to $30 \mathrm{mmHg}$. This resulted in decreased rSAP to $30 \mathrm{mmHg}$ (approximately $40 \%$ of pre-clamping systemic BP).

\section{Condition 4}

When FA perfusion was restarted, rSAP recovered to the value in Condition 1 . We identified distal perfusion as very important to the supply of collateral flow to the spinal cord.

Changes in rSAP under each condition revealed that collateral flow from the upper and lower body to SAs within the clamped region was sufficient to avoid SCII, and a collateral network existed between the reconstructed SA and surrounding SAs. These findings indicated that additional SA reconstruction was unnecessary.

After the essential SA was reconstructed, the graft prosthesis was anastomosed to the main graft and perfused by moving the clamp position distally. After securing a more stable blood supply to the spinal cord, rewarming was initiated and distal anastomosis was performed. When tympanic temperature exceeded $25^{\circ} \mathrm{C}$, MEPs appeared, and their amplitude increased to $80 \%$ of the initial value by the end of surgery. The patient was extubated two days after surgery and was discharged without any neurological deficits on day 28 after surgery. Contrast-enhanced computed tomography performed before discharge showed a well-reconstructed Th10 right segmental artery, with markedly developed collaterals.

\section{Discussion}

In surgery for TAAA and the descending aorta, the need for SA reconstruction remains controversial, with discussions differing among institutions. ${ }^{3)}$ However, many reports have recommended reconstructing SAs within the clamped region to prevent SCII. ${ }^{1,4,5)}$ Our strategic method for preventing SCII is to reconstruct SAs, even when the AKA is not identified, because a collateral network exists among SAs. $\left.{ }^{6}\right)$ The reconstruction of SAs is time-consuming when ensuring patency of the reconstructed graft prosthesis. Another concern is the risk of SCII during SA reconstruction, which may be resolved by SA perfusion. Under normothermic CPB, MEPs may disappear or decrease in amplitude, which is very stressful to surgeons. Deep hypothermia could be essential to resolving these problems because perfusion would be unnecessary, providing sufficient time for SA reconstruction. ${ }^{7,8)}$

Application of MEPs is a useful method for intraoperatively evaluating spinal cord ischemia during normothermic or mildly hypothermic TAAA surgery. ${ }^{1}$ However, because the amplitude of MEPs decreases and disappears in surgeries performed under deep hypothermia, spinal cord function after ischemia during aortic clamping cannot be assessed under those circumstances. ${ }^{2)}$ Consequently, the presence of SCII must be checked after rewarming following graft replacement, risking delays to appropriate 
treatments.

The present case used an rSAP monitoring method to evaluate spinal cord circulation to substitute for MEPs that disappear under deep hypothermia. This method estimates SCBF from the rSAP after opening the aortic aneurysm and is based on previously reported results from basic animal research. $\left.{ }^{9}\right)$ We clarified the correlations between mean systemic BP, segmental arterial pressure (SAP), and SCBF in the animal study. We found that, even under extensive SA clamping, the spinal cord blood supply via collateral networks remains functional, and SAP is highly and positively correlated with SCBF. Based on a single regression equation, maintaining SAP at 50\%-60\% of the pre-clamping systemic BP was estimated as necessary to ensure the pre-clamping SCBF.

Etz et al. measured spinal cord perfusion pressure (SCPP) for $24 \mathrm{~h}$ postoperatively in clinical cases, using a pressure catheter placed in the SA of the aortic replacement region, and reported that patients who developed postoperative neurological disorders showed significantly decreased SCPP $\left(<40 \%\right.$ of systemic BP). $\left.{ }^{10}\right)$

In the present case, SAP was measured after reconstruction of major SA, and a pressure of $60 \%-70 \%$ of the preclamping systemic BP was observed. We were, therefore, able to determine that SCBF via collateral flow was sufficient. Furthermore, inducing a "vascular steal phenomenon" of the backflow from the SAs (Condition 2), and decreasing the rate of perfusion from the FA (Condition 3), led to a significant decrease in rSAP. This confirmed the presence of an abundance of collateral networks to the spinal cord SA within the aortic clamping region.

When rSAP is low, the following approaches are effective for increasing SCBF via collateral networks: 1) increasing systemic BP with increased doses of vasopressors, such as NOA; or 2) increasing the flow through cardiopulmonary bypass. On the other hand, when rSAP is persistently $\leq 50 \%$ of systemic BP and does not increase with various approaches, other measures may be important, such as 1) promptly initiating antegrade perfusion from the reconstructed SA; or 2) considering additional $\mathrm{SA}$ reconstruction.

\section{Conclusion}

Intraoperative rSAP measurement offers a means of estimating the status of spinal cord circulation during aortic clamping under deep hypothermia, even when MEPs do not function.

\section{Disclosure Statement}

All authors have no conflicts of interest to declare.

\section{Additional Note}

Written informed consent was obtained from the patient.

\section{Author Contributions}

Writing: YK

Critical review and revision: all authors

Final approval of article: all authors

Accountability for all aspects of the work: all authors

\section{References}

1) Jacobs MJ, Mess W, Mochtar B, et al. The value of motor evoked potentials in reducing paraplegia during thoracoabdominal aneurysm repair. J Vasc Surg 2006; 43: 239-46.

2) Shinzawa M, Yoshitani K, Minatoya K, et al. Changes of motor evoked potentials during descending thoracic and thoracoabdominal aortic surgery with deep hypothermic circulatory arrest. J Anesth 2012; 26: 160-7.

3) Etz CD, Halstead JC, Spielvogel D, et al. Thoracic and thoracoabdominal aneurysm repair: is reimplantation of spinal cord arteries a waste of time? Ann Thorac Surg 2006; 82: 1670-7.

4) Afifi RO, Sandhu HK, Zaidi ST, et al. Intercostal artery management in thoracoabdominal aortic surgery: to reattach or not to reattach? J Thorac Cardiovasc Surg 2018; 155: 13728.e1.

5) Kuniyoshi Y, Koja K, Miyagi K, et al. Prevention of postoperative paraplegia during thoracoabdominal aortic surgery. Ann Thorac Surg 2003; 76: 1477-84.

6) Uezu T, Koja K, Kuniyoshi Y, et al. Blood distribution to the anterior spinal artery from each segment of intercostal and lumbar arteries. J Cardiovasc Surg (Torino) 2003; 44: 63745.

7) Kouchoukos NT, Masetti P, Rokkas CK, et al. Safety and efficacy of hypothermic cardiopulmonary bypass and circulatory arrest for operations on the descending thoracic and thoracoabdominal aorta. Ann Thorac Surg 2001; 72: 699707; discussion, 707-8.

8) Kise Y, Kuniyoshi Y, Ando M, et al. Transapical aortic perfusion using a deep hypothermic procedure to prevent dissecting lung injury during re-do thoracoabdominal aortic aneurysm surgery. J Cardiothorac Surg 2017; 12: 32.

9) Kise Y, Kuniyoshi Y, Inafuku H, et al. Directly measuring spinal cord blood flow and spinal cord perfusion pressure via the collateral network: correlations with changes in systemic blood pressure. J Thorac Cardiovasc Surg 2015; 149: 360-6.

10) Etz CD, Di Luozzo G, Zoli S, et al. Direct spinal cord perfusion pressure monitoring in extensive distal aortic aneurysm repair. Ann Thorac Surg 2009; 87: 1764-73; discussion, 1773-4. 